CLAWAR 2020: 23rd International Conference on

Climbing and Walking Robots and the Support

Technologies for Mobile Machines,

Moscow, Russian Federation, 24-26 August 2020.

https://doi.org/10.13180/clawar.2020.24-26.08.46

\title{
3D Reconstruction of Historical Sites using an UAV
}

\author{
P. Silva, A. Dias, A. Pires. T. Santos, A. Amaral, P. Rodrigues, J. Almeida and E. Silva \\ Centre for Robotics and Autonomous Systems, INESC TEC, \\ 431 Rua Dr. António Bernardino de Almeida, Porto, 4200-072, Portugal \\ E-mail: pedro.a.peixoto, andre.dias, ana.c.pires, tiago.a.santos, alexandre.a.oliveira, paulo.m.rodrigues, \\ jose.m.almeida and eduardo.silva@inesctec.pt \\ www.inesctec.pt
}

\begin{abstract}
This paper addresses Three-Dimensional (3D) reconstruction of historical sites with an Unmanned Aerial Vehicle (UAV), combining the information from a visible spectrum camera with a Light Detection and Ranging (LiDAR). The developed solution was validated in two sites located in Monastery of Tibães (Braga, NW Portugal), within the scope of MineHeritage project, which intends to reach society on the importance of raw materials through a historical approach. The outputs obtained from the datasets, resulted in a successfully 3D reconstruction of the two studied sites on the Monastery. Although the research is still ongoing on this topic, this paper is a starting point and an important contribution to this field and this type of scenarios
\end{abstract}

\section{Introduction}

In recent years, the documentation of heritage sites has gained considerable importance, ${ }^{1}$ especially sites threatened by a set of unpredictable factors, such as war, natural disasters, climate change, vandalism, and aging. ${ }^{2}$ Besides, heritage sites have a crucial role in the development of human beings ${ }^{3}$ and they should be preserved. 3D reconstruction of historical sites has become increasingly fundamental and necessary due to various reasons, such as preservation, reconstruction, and as-built documentation. ${ }^{4}$ The MineHeritage project was developed to address this need using educational tools to reach society on the importance of raw materials, cultural heritage, and ancient mining through an historical approach.

During the last decade, the development of UAVs has been growing rapidly. ${ }^{5}$ In the past, they were designed primarily for military goals and applications. ${ }^{6}$ However, they have many advantages, such as low-cost, flexibility, high precision and capacity for data acquisition that makes UAVs a powerful tool nowadays. ${ }^{7}$ As a consequence, they have a huge variety of applications, such as search and rescue operations, construction and infrastructure inspection, precise agriculture, surveillance, among others. ${ }^{8}$ The cost-effectiveness and advantages of UAVs make them suitable platforms to survey historical heritage sites.

The two main approaches applied to perform 3D reconstruction with an UAV are: (1) LiDAR sensor; and (2) photogrammetry technique which uses a digital camera. ${ }^{7}$ LiDAR provides information about the $3 \mathrm{D}$ structure of the environment, in the form of a point cloud based on the distances measured by the light beams. The main advantages of LiDAR are the high precision, even at long distances, and the invariance to the lighting conditions. ${ }^{9}$ However, it does not provide visual information (color and texture) of the environment. ${ }^{10}$ Photogrammetry generates 3D color point clouds by using Structure from Motion (SfM) technique. ${ }^{11}$ Furthermore, photogrammetry requires specific weather conditions, it cannot penetrate a tree's canopy or map complex structures, such as power lines, taking a lot of time to process the data.

These two previous approaches are decoupled, i.e., they do not combine LiDAR with vision. Therefore, combining the functionalities of LiDAR with a vision system will allow the production of an accurate 3D point cloud, where each laser beam can be filled with the color acquired by the visible spectrum camera. 
The remainder of this paper is organized as follows. The next section introduces the MineHeritage project. Section 3 describes the developed methodology to perform 3D reconstruction, using a LiDAR and a visible spectrum camera. The results are presented in Section 4 and conclusions are placed, as well as the future work, in Section 5.

\section{MineHeritage Project}

This research was developed within the scope of MineHeritage Project funded by European Institute of Innovation and Technology (EIT) Raw Materials. MineHeritage intends to reach society on the importance of raw materials through a historical approach. Using Europe as a common foreground, where raw materials have been explored and traded through times, this project shows that technological developments increased the demand for certain raw materials linking regions to social strategies of work and progress. Through the history of mining, this work will show the importance of raw materials in the development of Europe, European culture and society. In particular, this Wider Society Learning project intends to use cultural heritage, mining and raw materials to create popular educational tools for the dissemination of raw materials importance to society through historic periods. Moreover, by applying an UAV approach it will be possible to acquire several datasets to provide different outputs for the project.

The importance of digitally record, archive and document significant cultural and historical heritage is increasing from a worldwide perspective. Organizations such as $\mathrm{CyArk}^{12}$ and OpenHeritage3D portal ${ }^{13}$ provide open access to $3 \mathrm{D}$ cultural heritage datasets for several purposes, such as: education, research and other non-commercial uses. Presently, cultural heritage is at risk due to several threats, from natural disasters to human conflict, urbanization or the effects of climate change. The gathered and stored data can serve as a point in time archives.

The scope of MineHeritage will also address the future integration of different datasets in OpenHeritage3D portal, accessible and open worldwide. The research presented here highlights the geo-mining heritage for future conservation, recovery, and discovery, as well as other scientific and technological studies concerning the application of UAVs or other types of methods to support $3 \mathrm{D}$ reconstruction.

\section{3D Reconstruction Methodology}

The high-level software architecture to perform 3D reconstruction combining LiDAR and camera is displayed in Fig. 1. This pipeline is divided in two layers which were developed using Robot Operating System (ROS) framework.

The first layer is done offline, while the second one in real-time. The offline layer comprises the extrinsic calibration process between a LiDAR and a camera. The second layer is composed by several stages, as shown in Fig. 1. LiDAR raw data is converted into a point cloud in the sensor frame. These points are converted into the camera frame, through extrinsic parameters, and then to the image plane. After this stage, each point is associated with the corresponding color, through the Red-Green-Blue (RGB) component of the image, obtaining a color point cloud.

The LiDAR pose can have a fixed relation with respect to the UAV, if attached to the platform, or a dynamic one, if connected to a gimbal, for example. UAV pose is estimated from a Real-Time Kinematic (RTK) Global Navigation Satellite System (GNSS) receiver and an Inertial Measurement Unit (IMU). The robot pose will allow, together with the color point cloud and LiDAR pose, to perform the $3 \mathrm{D}$ reconstruction of the environment that UAV has mapped.

In the next sections the notation italic, boldfaced lowercase, and boldfaced uppercase 


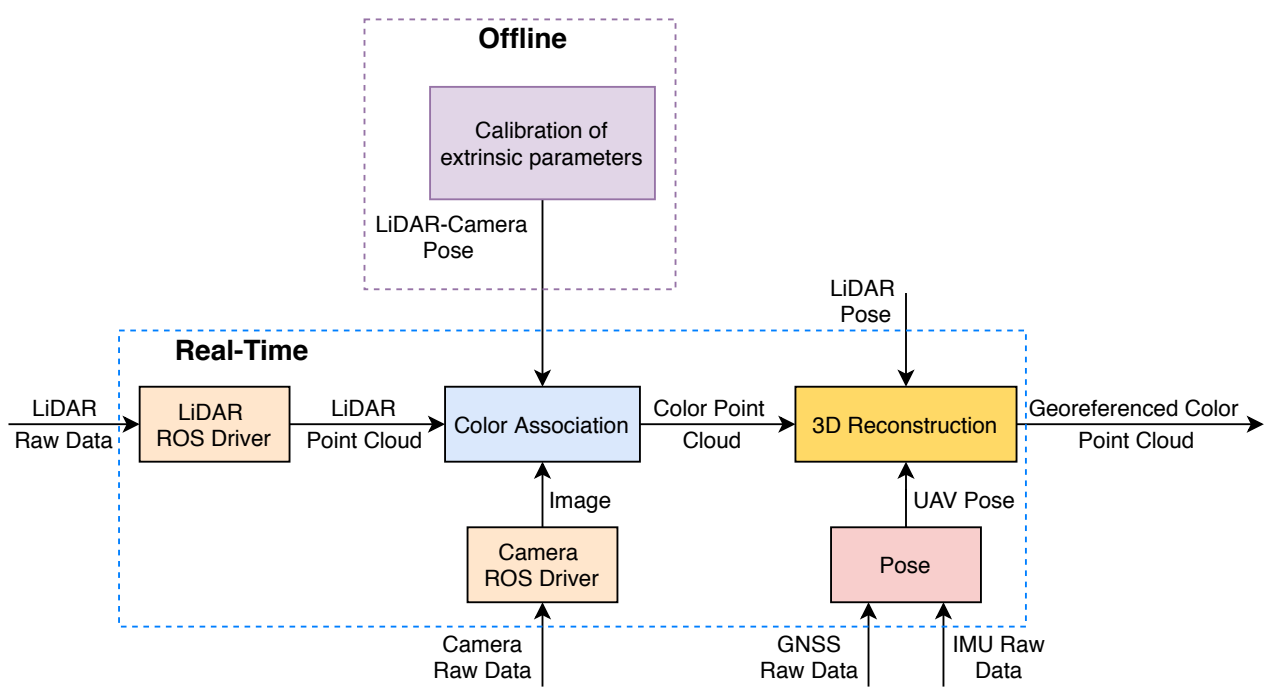

Fig. 1: High-level LiDAR-Camera 3D Reconstruction Pipeline

letters represent scalars, vectors, and matrices, respectively.

\subsection{Automatic Extrinsic Calibration LiDAR-Camera}

The problem of extrinsic calibration of a LiDAR and a camera is to estimate the rotation and the relative translation between these two sensors. The implemented method is based on the work developed by Zhou et al. ${ }^{14}$ and can be used in all visible spectrum cameras and 3D LiDARs. The authors use plane and line correspondences to obtain the extrinsic parameters.

In this method, is used a checkerboard as the calibration target. The target plane and its four boundaries are exploited to estimate the rotation $\mathbf{R}_{L}^{C}$ and the translation $\mathbf{t}_{L}^{C}$ between LiDAR and camera. Given $N$ target pose, it is possible to estimate the plane and line parameters in the camera and LiDAR frame. The major advantage of this method is that it reduces the number of poses required to one.

The method is illustrated in Algorithm 3.1.

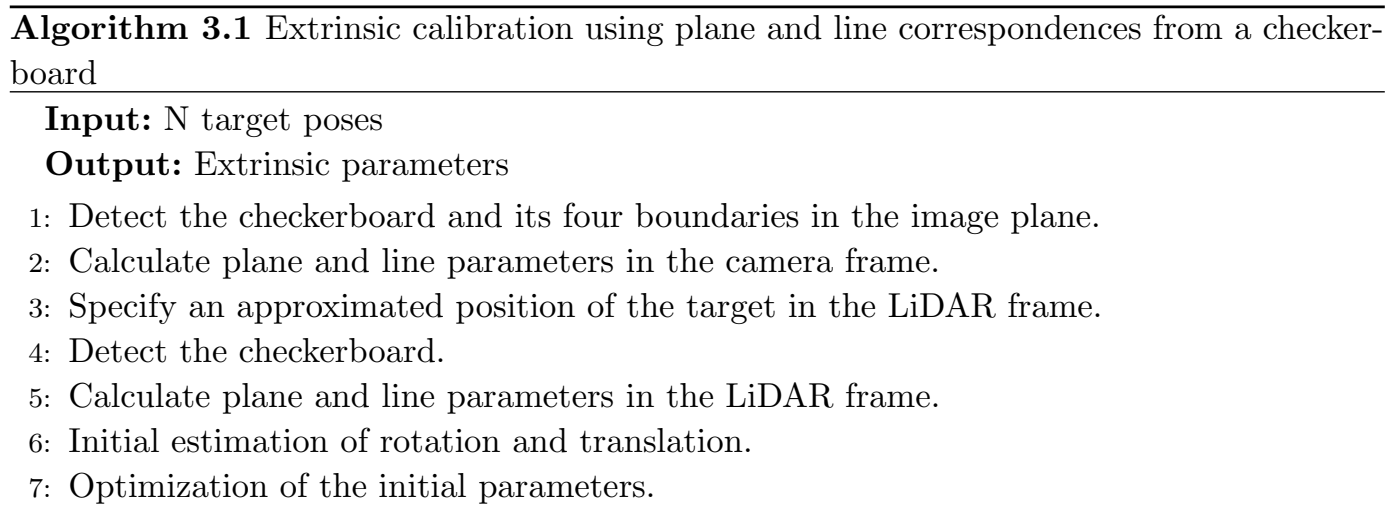




\subsection{Colorizing point cloud}

After calibrating the extrinsic parameters between the LiDAR and the camera, it is possible to transform the 3D laser points into the camera frame. Assuming that LiDAR and camera share the same principal axis, objects measured by the laser can be visible by the camera. ${ }^{15}$ Therefore, the color of each point can be determined from a single observation, that is, based on the closest frame between the two sensors.

In order to colorize the point cloud, it is important that both sensors are synchronized (same trigger and/or clock source). The GNSS receiver allows us to synchronize all timestamps of LiDAR and camera data, using Coordinated Universal Time (UTC) as a reference. The receiver sends GNSS data and a Pulse Per Second (PPS) that are used to synchronize directly the LiDAR data, as well as the Central Processing Unit (CPU) clock. The camera is hardware triggered by the onboard computer, in which the trigger timestamp is stored and associated with the respective image.

The LiDAR points in the camera frame can be obtained by Eq. (1).

$$
\mathbf{P}^{C}=\mathbf{R}_{L}^{C} \mathbf{P}^{L}+\mathbf{t}_{L}^{C}
$$

Generally, a 3D LiDAR has a much larger Field of View (FoV) than a perspective projection camera. The laser points outside of camera FoV must be discarded. The condition given in Eq. (2) eliminates LiDAR points that are behind the camera.

$$
Z^{C}>0
$$

The points in camera frame can be converted to the image by the camera intrinsic matrix A (see Eq. 3). Before projecting the points, it is necessary to remove the image distortion. Possibly, some points will be discarded by Eq. (4).

$$
\begin{gathered}
{\left[\begin{array}{l}
u \\
v \\
1
\end{array}\right]=\mathbf{A}\left[\begin{array}{l}
x^{C} \\
y^{C} \\
z^{C}
\end{array}\right]} \\
0 \leq u<w \\
0 \leq v<h
\end{gathered}
$$

The image size is equal to $[w, h]$ and $u$ and $v$ represent the $x$ and $y$ pixel of the image, respectively. The color of the 3D LiDAR points is given by the RGB value of each pixel.

\subsection{D Reconstruction}

To perform 3D reconstruction with centimeter accuracy, it is necessary to use RTK technique. As already stated, UAV position and orientation are estimated from a dual-band GNSS receiver and an IMU, resulting in a pose with high precision (centimeter accuracy).

The UAV localization will make possible to join the various point clouds. The result will be a georeferenced color point cloud of the environment that has been mapped. For that, it is fundamental to analyse all coordinate systems involved in the robot system. Figure 2 illustrates all frames of the UAV system.

World coordinates are represented in a local referential, also called Local Tangent Plane (LTP). This referential consists of a tangential flat surface at a point on the Earth, where the robot's navigation takes place. The local referential used is East-North-Up (ENU), where the $x$ axis indicates East, $y$ North and $z$ Up.

After knowing all reference frames involved, it is possible to convert LiDAR points to world frame through Eq. (5), using Homogeneous transformations.

$$
\left[\begin{array}{c}
\mathbf{P}^{W} \\
1
\end{array}\right]=\mathbf{T}_{b}^{W} \mathbf{T}_{L}^{b}\left[\begin{array}{c}
\mathbf{P}^{L} \\
1
\end{array}\right]
$$




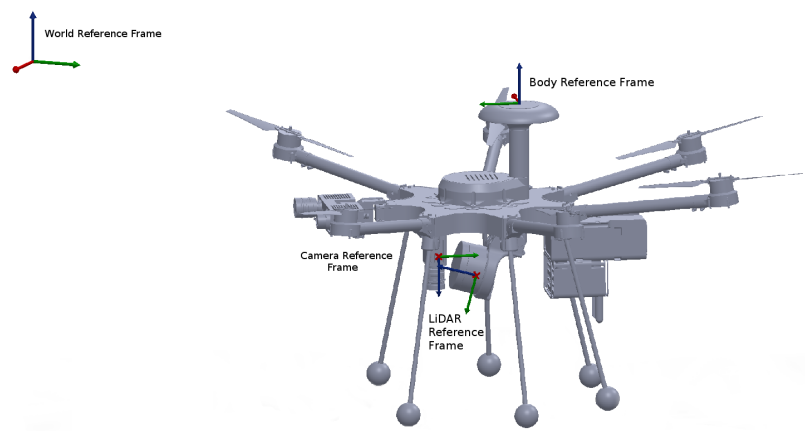

Fig. 2: STORK I UAV - Coordinate system of the body, LiDAR, camera and world ( $x$ axis is represented in red, $y$ in green and $z$ in blue).

where $\mathbf{T}_{L}^{b}$ represents the transformation between LiDAR and body frame, $\mathbf{T}_{b}^{W}$ represents the transformation between body and world frame and $\mathbf{P}^{L}$ a point in LiDAR frame.

\section{Results}

\subsection{Experimental Setup}

STORK $^{16}$ (Fig. 3a) is a hexacopter autonomous UAV and its primary application is the power assets inspection. However, it has been used in other applications, such as search and rescue operations, 3D mapping, environmental monitoring, surveillance, and patrol.

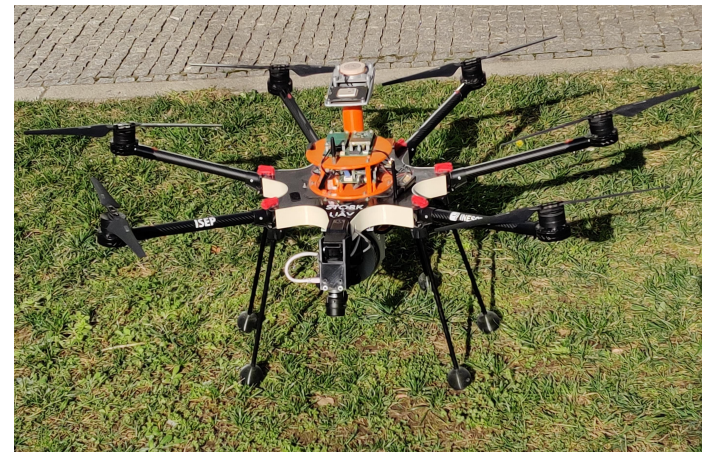

(a)

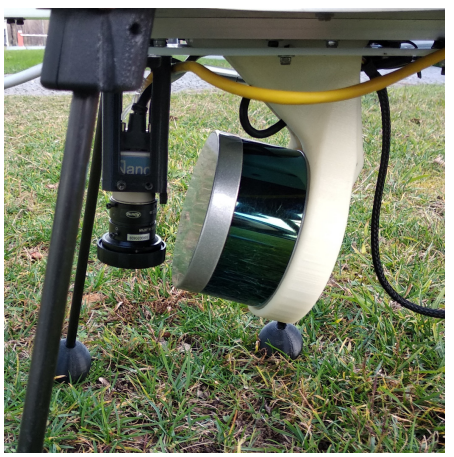

(b)

Fig. 3: STORK UAV general view (a); LiDAR and camera coupled in STORK UAV (b).

The UAV has several sensors integrated, namely perception and navigation sensors. For navigation, it has a high-precision IMU and a dual-band GNSS receiver, alongside the default low-cost sensors of autopilot. In terms of environmental perception, it has a 3D LiDAR (Velodyne VLP-16), two visible spectrum cameras (a fixed Teledyne Dalsa G3GC10-C2450, pointing down, and a FLIR Point-Grey Chameleon3, connected to a gimbal) and, in some applications, a thermographic camera. Figure $3 \mathrm{~b}$ illustrates LiDAR and camera coupled in STORK UAV.

\subsection{Datasets}

The datasets were performed in the stairway (Fig. 4a) and enclosure (Fig. 4b) of the Monastery of Tibães (Braga, NW Portugal), one of the studied sites within the scope of 
MineHeritage project. This monastery was founded in the late 11th century and was the mother house of the Order of Saint Benedict for Portugal. Most of the magnificent construction dates to the 17 th and 18 th centuries. Despite the degradation of the monastery over the last few decades, the Portuguese Government acquired it and began its heritage protection to the current day.

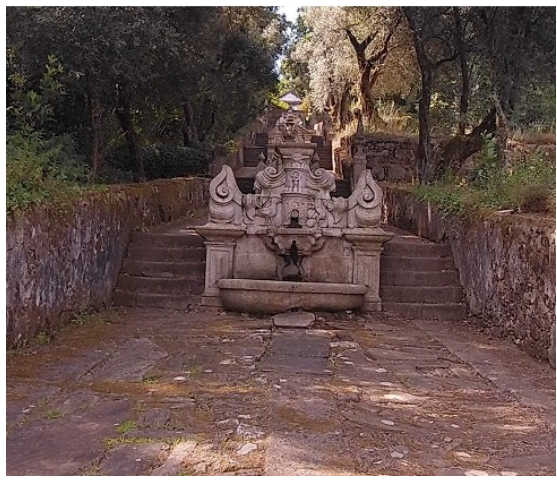

(a)

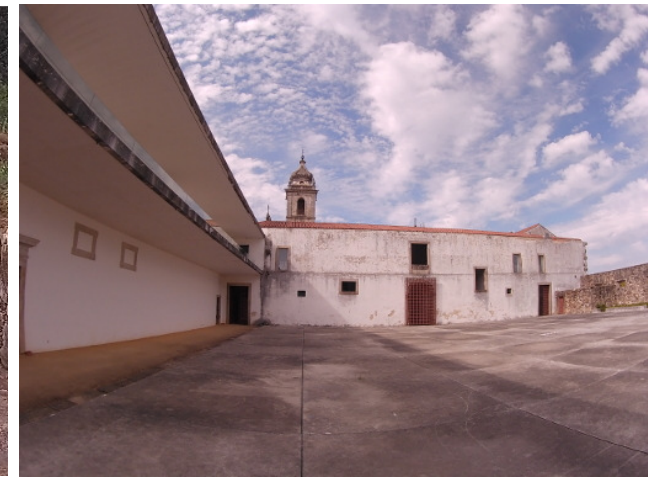

(b)

Fig. 4: Stairway (a) and enclosure of the Monastery of Tibães (b).

The stairway is mainly composed of several water sources, that are interspersed with stairs, landings, and trees. On the other hand, the enclosure is mainly formed of buildings and vegetation. Unlike the stairway, this zone is more wide and the UAV localization can be more precise (GNSS signals are not obstructed, therefore are more consistent and stronger).

The UAV started by mapping a field of vegetation near the staircase, making a straight line to obtain a high-quality initialization of its pose. After this, it moved to the first level of the staircase and went up among the trees to the last level located at the top. The first water source is illustrated in Fig. 5a from UAV onboard camera, Fig. 5b represents LiDAR raw point cloud (color given by LiDAR intensity) and Fig. 5c illustrates LiDAR points projected in image plane, using the extrinsic parameters.

These pixels were used in the color association process of each LiDAR point. The camera's FoV is much lower than LiDAR's and, therefore, the color point cloud (Fig. 5d) has a lower number of points compared to the raw point cloud (Fig. 5b). The LiDAR used (Velodyne VLP-16) has 16 laser beams which are all viewed by the camera.

In the second flight, the procedure was the same as done in the stairway. Fig. $6 \mathrm{a}$ and $6 \mathrm{~b}$ shows a water tank in the enclosure area and a bottom view of the stairway, respectively. These two point clouds are georeferenced and they can be combined into a unique color point cloud (Fig. 7).

From this figure, it is possible to verify a color difference in the vegetation zone (intermediate area of Fig. 7). This difference is obtained mainly because the camera used is sensitive to infrared. To solve this problem, it is necessary to add a Infrared Radiation (IR) bandpass filter. This filter will block the light outside the visible range and let visible light pass.

\section{Conclusion and Future Work}

In this paper, we present a 3D reconstruction methodology combining a 3D LiDAR and a visible spectrum camera. We validate the developed approach along two datasets in the Monastery of Tibães, a historical and heritage mining site located in Braga (NW Portugal). Comparing the color point cloud obtained with the original images, it is possible to conclude 


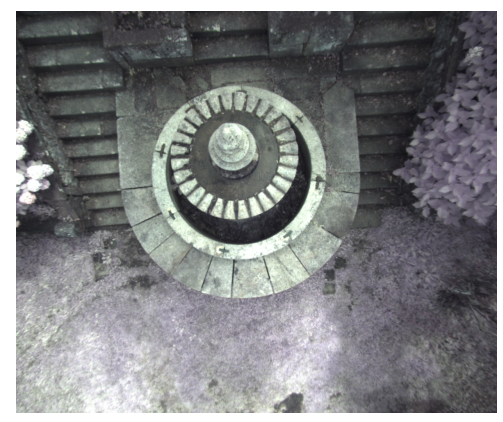

(a)

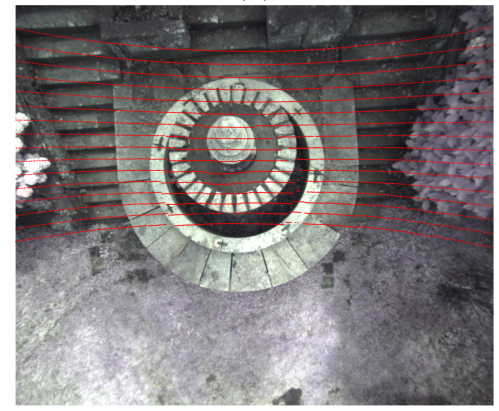

(c)

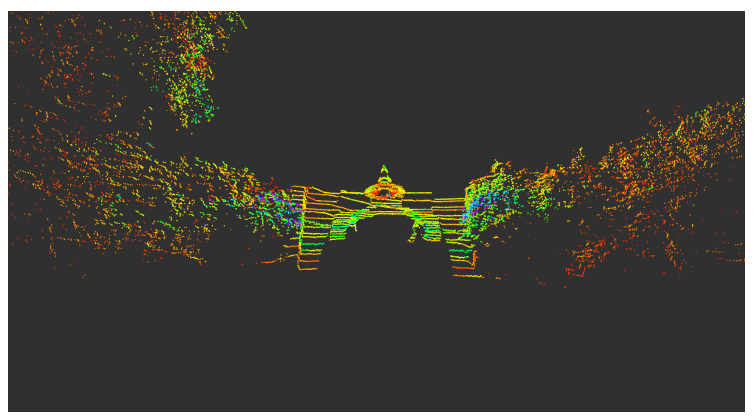

(b)

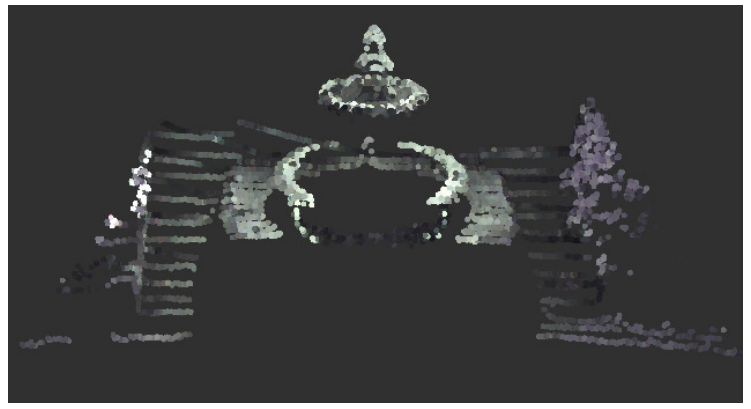

(d)

Fig. 5: First water source (a); LiDAR raw point cloud (b); LiDAR points projected in image plane (c); Color point cloud of the first water source (d).

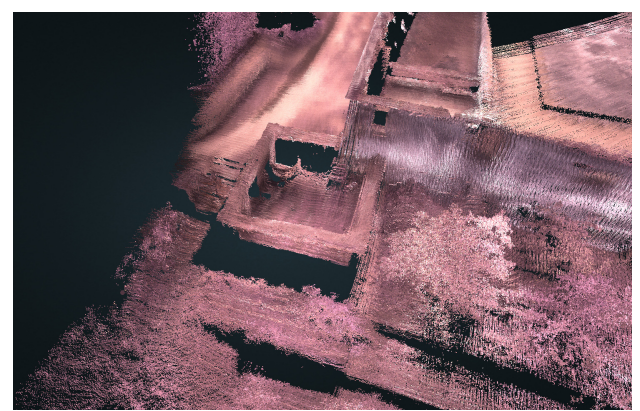

(a)

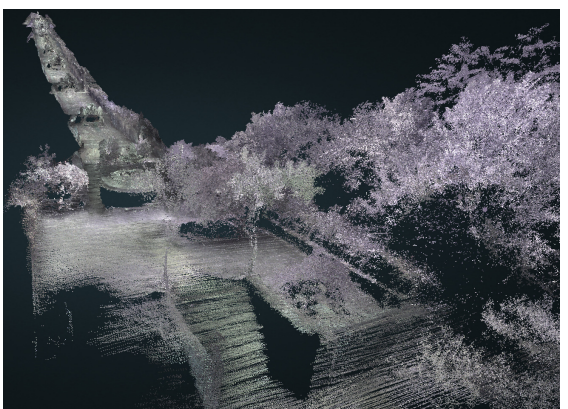

(b)

Fig. 6: Water tank in the enclosure area (a); Bottom view of the enclosure of the Monastery of Tibães (b).

that the color association has a strong quality and that the UAV 3D reconstruction has been successfully achieved.

In terms of future work, it is important to integrate all the information provided by the camera downward near to the LiDAR and also the camera available in the gimbal, into the $3 \mathrm{D}$ reconstruction. This integration of more cameras in the processing pipeline of the 3D reconstruction will certainly allow to address other application scenarios, such as: inspection of power lines and gas pipelines, where it is necessary to have 3D reconstruction not only from top to bottom, but also in both sides of the UAV trajectory. The development of an algorithm to perform a correct alignment between point clouds, will also be a line of research. 


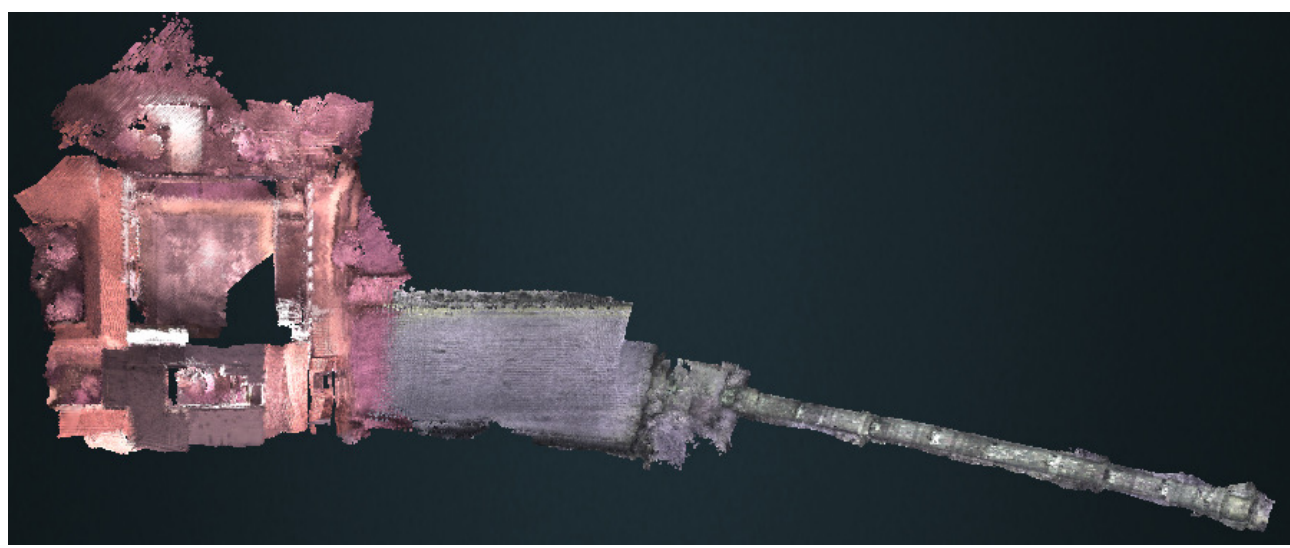

Fig. 7: Top view of the stairway and the enclosure of the Monastery of Tibães.

\section{Acknowledgments}

This work is funded by the MineHeritage Project (EIT/RAW MATERIALS/SGA2019/1) funded by EIT Raw Materials [Nr. 18111; Main Theme 1 Exploration and raw materials resources assessment; Area D3 RM Academy; Segment D3.1 Wider Society Learning.

\section{References}

1. A. Murtiyoso and P. Grussenmeyer, The Photogrammetric Record 32, 206 (2017).

2. F. Hassani, International Archives of the Photogrammetry, Remote Sensing 8 Spatial Information Sciences 40 (2015).

3. H. M. Yilmaz, M. Yakar, S. A. Gulec and O. N. Dulgerler, Journal of Cultural Heritage 8, 428 (2007).

4. Z. Xu, L. Wu, Y. Shen, F. Li, Q. Wang and R. Wang, Remote sensing 6, 10413 (2014).

5. C. F. Liew, D. DeLatte, N. Takeishi and T. Yairi, arXiv preprint arXiv:1711.10085 (2017).

6. F. Nex and F. Remondino, Applied geomatics 6, 1 (2014).

7. H. Ren, Y. Zhao, W. Xiao and Z. Hu, International Journal of Coal Science ES Technology 6, 320 (2019).

8. H. Shakhatreh, A. H. Sawalmeh, A. Al-Fuqaha, Z. Dou, E. Almaita, I. Khalil, N. S. Othman, A. Khreishah and M. Guizani, IEEE Access 7, 48572 (2019).

9. Z. Pusztai and L. Hajder, Accurate calibration of LiDAR-camera systems using ordinary boxes, in Proceedings of the IEEE International Conference on Computer Vision, 2017.

10. S. Verma, J. S. Berrio, S. Worrall and E. Nebot, Automatic extrinsic calibration between a camera and a 3d lidar using 3d point and plane correspondences, in 2019 IEEE Intelligent Transportation Systems Conference (ITSC), 2019.

11. M. J. Westoby, J. Brasington, N. F. Glasser, M. J. Hambrey and J. M. Reynolds, Geomorphology 179, 300 (2012).

12. Cyark. https://www.cyark.org/, [Online; accessed 15 February 2020].

13. OpenHeritage3D. https://openheritage3d.org/, [Online; accessed 15 February 2020].

14. L. Zhou, Z. Li and M. Kaess, Automatic extrinsic calibration of a camera and a 3D LiDAR using line and plane correspondences, in 2018 IEEE/RSJ International Conference on Intelligent Robots and Systems (IROS), 2018.

15. P. Vechersky, M. Cox, P. Borges and T. Lowe, IEEE Robotics and Automation Letters , 3575 (2018).

16. F. Azevedo, A. Dias, J. Almeida, A. Oliveira, A. Ferreira, T. Santos, A. Martins and E. Silva, Sensors 19(04 2019). 